districts had a modest impact on FSW/client HIV prevalence if duration exceeded 9 years (Abstract P2-S2.16 figure 1), independently of client duration. The impact of FSW duration below 3 years on FSW/client HIV was critical. FSW/client HIV prevalence was more sensitive to variation in client duration exceeding 9 years (Abstract P2-S2.16 figure 1)

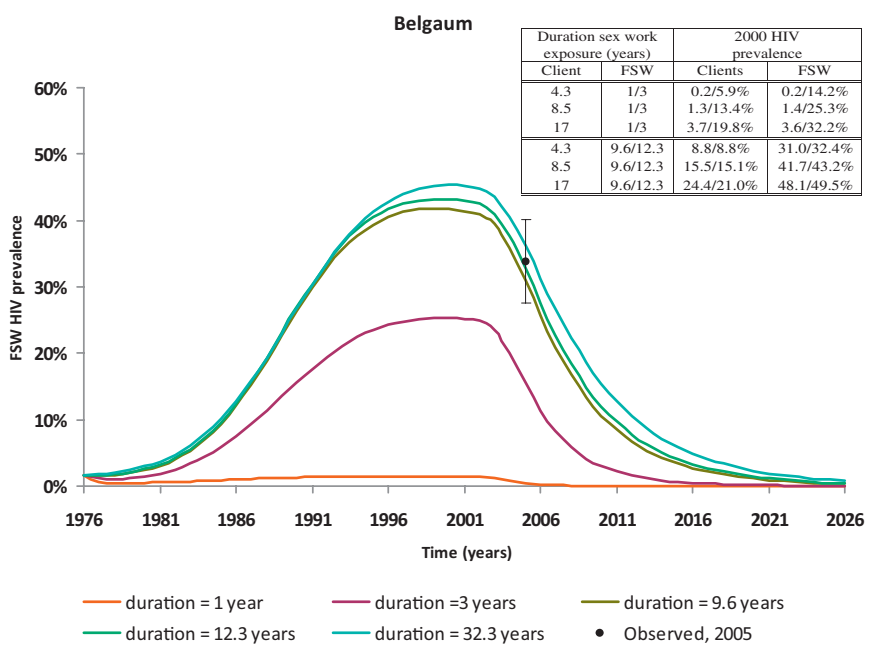

Abstract P2-S2.16 Figure 1

Conclusions Crude duration may underestimate FSW duration by up to $32 \%$. This level of error is unlikely to influence FSW HIV prevalence projections. Large variation in HIV across districts is unlikely to be explained by FSW duration alone if exceeding 9 years but could be potentially be explained by variation in client duration.

\section{P2-S2.17 COMMUNITY-LED STRUCTURAL INVENTION'S PROMISE FOR HIV PREVENTION: A CASE STUDY FROM THE ASHODAYA SAMITHI SEX WORKER COLLECTIVE OF MYSORE, INDIA.}

doi:10.1136/sextrans-2011-050108.313

${ }^{1} \mathrm{~N}$ O'Brien, ${ }^{2} \mathrm{~S}$ Reza-Paul, ${ }^{3} \mathrm{P}$ Akram, ${ }^{3} \mathrm{~S}$ Jai, ${ }^{3} \mathrm{~K}$ T Venukumarc, ${ }^{3} \mathrm{M} \mathrm{S}$ Venugopalc, ${ }^{3} \mathrm{M}$ P Fatima, ${ }^{2} \mathrm{R}$ Lorway. ${ }^{1} \mathrm{BC}$-Centre for Excellence in HIVIAIDS, Vancouver, Canada; ${ }^{2}$ Department of Community Health Sciences, University of Manitoba, Winnipeg, Canada; ${ }^{3}$ Ashodaya Samiti, India

Background As evidence has revealed the role of broader political, economic and local environmental factors in shaping HIV epidemic transmission patterns, new approaches have emerged that move beyond addressing individual risk behaviour to addressing the structural forces that condition risk. Within this context, Community-Led Structural Interventions (CLSI) aimed at reducing sex workers' vulnerability have demonstrated successes in reducing STI and HIV transmission in India.

Methods A comprehensive CLSI, know as Ashodaya Samithi, was initiated in the South Indian district of Mysore. Established in 2004 by the India AIDS Initiative (Avahan), Ashodaya is funded through the Bill and Melinda Gates Foundation and implemented by Karnataka Health Promotion Trust. Four-years into its inception, the project lays claim to significant successes. The collective has reported a reduction in STIs, an increase in condom use, and the mobilisation of 1500 sex workers. Our research describes and analyzes sex workers' participation as it unfurled across various phases of collectivisation. Qualitative, community-based research methodologies were utilised including; 40 open-ended interviews, participant observations and focus groups. Drawing upon these findings we explored how a community of sex workers came to develop the ability to recognise, challenge and significantly alter an array of unequal power relations that shape HIV vulnerability in their everyday lives.

Results Our research demonstrates the promise CLSI projects hold for preventing the spread of HIV. Ashodaya's success depends on its ability to set new community norms, create a safer working environment, improve stakeholder relationships, and provide a supportive network. The interrelated processes of mobilisation, collectivisation, sex-worker identity, and political action, which transformed day-to-day vulnerability to HIV for Mysore sex workers demonstrates how complex HIV prevention remains.

Conclusions Findings from the Ashodaya collective offer valuable lessons for public health practice. The Ashodaya collective demonstrates how CLSI strategies can be employed to help transform the very vulnerabilities that endanger health and sustain STI and HIV transmission. The collective provides an example of the positive impact of CLSIs and provides evidence for strategies that steer away from individual or education based public health programs, to those that engage with structural inequities.

\section{P2-S2.18 ARE WE DOING ENOUGH TO COVER RISK OF STI/HIV TRANSMISSION FROM THE LOVERS “OF FEMALE SEX WORKERS (FSWS)?"}

doi:10.1136/sextrans-2011-050108.314

M Doddamane, P Bhattacharjee, D Rao. Karnataka Health Promotion Trust, Bangalore, India

Background Condoms use among Female sex workers with paid clients found to have increased rapidly in three years from $58 \%$ to $76 \%$ (IBBA data) due to scale up of interventions among them. However condom use with lovers still remains low at 33\% (IBBA 2008). PBS 2009 shows 50\% of the sex workers have lovers. Programmatically it has been challenging to design and implement programme to reach out to Lovers and to provide them services consistently. To understand the Lovers and the risk factor associated with them we conducted Focused Group Discussion (FGDs).

Method 14 Separate FGDs were conducted with both the FSWs and their Lovers in seven districts of Karnataka. A structured tool guided the Facilitators of the FGD. Each FGD was documented by a Recorder.

Results Three fourths of the FSWs had more than one lover at any given point of time. One fourth of the lovers had physical relationship with other FSWS or women other than wife. Half of the FSWs especially the urban based changed lovers in less than a year. Majority of the lovers knew that their partner is a sex worker. Key barriers for Lovers to use condoms with the sex workers were: 1. Faith and trust with FSWs (almost half of the respondents) 2. Condoms don't give pleasure (one third of the respondents). The barriers stated by the FSWs were: 1. Faith and trust in the lover because they are like husbands (one third of the respondents) 2. Did not want to make the lover suspicious (one fourth of the respondents) One fourth of the sex workers responded experiencing harassment from the lovers especially for money. Only one third of the lovers had correct information about HIV (modes of transmission) and negligible number of lovers accessed STI services.

Conclusion Evidence shows that there is clear need to intervene with Lovers of female sex workers to improve condom use among them. The interventions have to address not only gaps in information, condom use, access to service, but also the perception of faith and trust. 